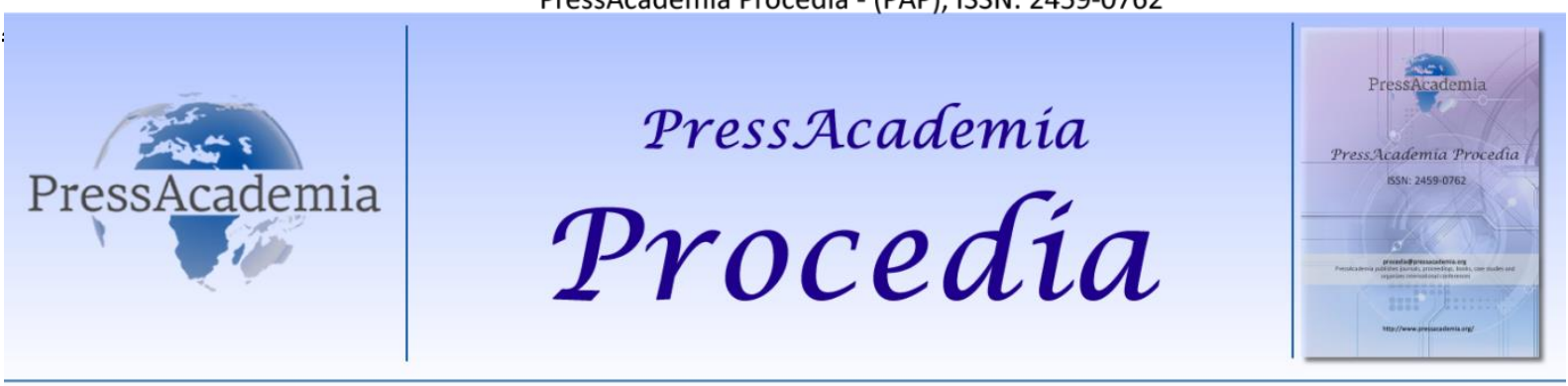

2nd World Conference on Technology, Innovation and Entrepreneurship

May 12- 14, 2017, Istanbul, Turkey. Edited by Sefer Şener

\title{
A SURVEY AUTOMATIC TEXT SUMMARIZATION
}

\section{DOI: 10.17261/Pressacademia.2017.591 PAP-WCTIE-V.5-2017(29)-p.204- 213}

\section{Oguzhan Tas ${ }^{1}$, Farzad Kiyani ${ }^{2}$}

Istanbul Sabahattin Zaim University, Computer Engineering Department. oguzhan.tas@std.izu.edu.tr Istanbul Sabahattin Zaim University, Computer Engineering Department. farzad.kiyani@izu.edu.tr

\begin{abstract}
Text summarization is compress the source text into a diminished version conserving its information content and overall meaning. Because of the great amount of the information we are provided it and thanks to development of Internet Technologies, text summarization has become an important tool for interpreting text information. Text summarization methods can be classified into extractive and abstractive summarization. An extractive summarization method involves selecting sentences of high rank from the document based on word and sentence features and put them together to generate summary. The importance of the sentences is decided based on statistical and linguistic features of sentences. An abstractive summarization is used to understanding the main concepts in a given document and then expresses those concepts in clear natural language. In this paper, gives comparative study of various text summarization techniques.
\end{abstract}

Keywords: Text summarization, natural language processing, NLP, statistical approach.

\section{INTRODUCTION}

A summary can be defined as a text that is produced from one or more texts, that contains a siginificant partion of the information in original text(s), and that is no longer than half of the original text(s) [1]. According to another reference, text summarization is the process of distilling the most important information from a source (or sources) to produce abridged version for a particular user (or users) and task (or tasks)[2].

Automatic Text summarization is a challenging problem and it gaining much significance these days. The automatic summarization means an automatically summarized output is given when an input applied. Although the research on the Automatic Text summarization has been started 1950's in IBM Research Laboratories[3], in the recent years the field of Text Summarization has experienced an exponential growth because of the Internet. It is very difficult to manually summarize large documents of the text because there is a huge amount of information on the Internet. On the other hand, Internet is a rich library that provides more information than is demanded. Therefore, it is crucial for searching relevant document through an huge number of documents available. The target of text summarization is shorten the source text into a diminished version conserving its information content and overall meaning.

Text summarization methods can be classified into extractive and abstactive summarization. Abstractive Summarization is a method for novel phrasing describing the content of the text which requires heavy machinery from natural language processing, including grammars and lexicons for parsing and generation. Extractive Summarization is a method for determining salient text units (typically sentences) by looking at the text unit's lexical and statistical relevance or by matching phrasal patterns [4] Abstraction approaches provide sophisticated summaries and adapt well to high compression rates while extraction approaches are easy to adapt larger sources although the resulting summaries may be incoherent. Extractive Summaries are formulated by extracting key text segments such as sentences or passages from the text, based on statistical analysis of individual or mixed surface level features[5].

There are two different groups of text summarization. Inducative and Informative. Indicative summarization gives the main idea of the text to the user. The length of this summarization is around 5 percent of the given text. The informative 
summarization system gives brief information of the main text. The length of the informative summary is around 20 percent of the given text [6].

Furhermore, summarization methods can be classified according to source which can be single or multiple document summarization. In single document summarization, only one document is provided for summary generation. It is a simple and earliest approach for summarization. Extractive and abstractive both summaries methods can be applied on single document summarization. Multi document summarization is also very important part of summarization. More than one information sources are provided for summary generation. Many web based clustering systems like news were inspired from multi document summary. However, task of multiple document summarization technique is more difficult and complex than single document techniques. The real aim is not only to remove redundancy and indentify correct text for summary but also to provide novelty and ensuring that final summary should be coherent and complete in itself. So it was a challenge for them to consider all the documents and relate the summary[7].

\section{PREPOROCESSING AND EXTRACTION FEATURES}

In this paper, we mention about single and multiple document Extractive Summarization techniques because the most of the current automated text summarization systems use extradiction methods. Extractive summarization process can be divided into three phases. First phase is Pre-Processing, second phase is Processing.

In Pre-processing phase, there are many techniques, such as part of speech(POS) tagging, stop word filtering and stemming.

\subsection{Part of Speech(POS) Tagging}

Part of Speech Tagging is the the process of grouping or specifying the words of text according to speech category such as noun, verbs, adverbs, adjectives etc. The POS tagging process can be implemented by many algorithms such as Hidden Markov Model and Viterbi.

Figure 1: Step by Step Text Summarization

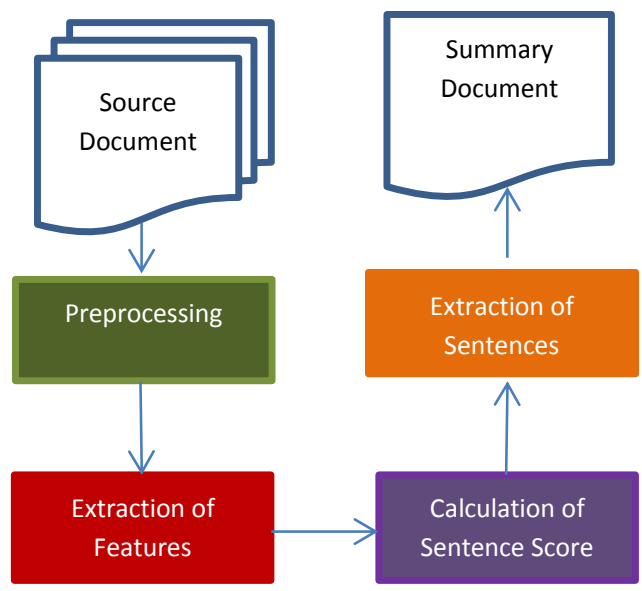

\subsection{Stop Word Filtering}

Stop words are words which are filtered out before or after processing of text(corpus), it is fully non-objective depends upon the situation. For example, a, an, in, by can be considered as a stop words and filtered from plain text.

\subsection{Stemming}

Stemming is the process of reducing inflected (or sometimes derived) words to their word stem, base or root form. For example, removing from -ed or -ing from verbs, using singular instead of plural noun, etc. There are many NLP tools which are using stemmer algorithms for executing the stemming.

\subsection{Feature Calculation}

In order to calculate the score of sentence $S$, indicating the degree whether it belongs to summary or not, the following feautures are generally used in the score of sentences. 


\subsubsection{Title Similarity}

A sentence has good score if it has maximum number of words similar in the title. The title similarity can be calculated by the number of words in the sentence that occur in the title and the total number of words in the title. The title similarity is defined as;

$$
\begin{aligned}
& \boldsymbol{f 1}=\frac{S \cap T}{t} \\
& \text { In formula; } \\
& S=\text { Set of words in sentence } \\
& T=\text { Set of words in title } \\
& S \cap T=\text { Similar words in sentence and title of document. }
\end{aligned}
$$

\subsubsection{Sentence Position}

In this feature, a sentence is evaluated by its position in the text. Whether it is the first 5 sentences in the paragraph, sentence position in text gives the importance of the sentences. We consider the first 5 sentences in the paragraph. This feature score is calculated by[6, 12].

$$
\begin{aligned}
f 2= & 5 / 5 \text { for } 1^{\text {st }}, 4 / 5 \text { for } 2^{\text {nd }}, 3 / 5 \text { for } 3^{\text {rd }}, \\
& 2 / 5 \text { for } 4^{\text {th }}, 1 / 5 \text { for } 5^{\text {th }}, \\
& 0 / 5 \text { for other sentences. }
\end{aligned}
$$

On the other hand, simple and different formula is used when calculating sentence position value[13].

$$
\begin{aligned}
& f 2=1, \text { if sentence is the beginning sentence in the text, } \\
& f 2=0, \text { if sentence is the middle of paragraphs in the text, } \\
& f 2=1 \text {, if sentence is the the last position in the text. }
\end{aligned}
$$

\subsubsection{Term Weight(Term frequency)}

The total term weight is calculated by computing $t f$ and idf for document. Here idf refers to inverse document frequency which simply tells about whether the term is common or rare across all documents[12,13]. The score of important score $w_{i}$ of word $i$ can be calculated by the traditional $t f . i d f$ methods.

$w_{i}=t f_{i} \cdot i d f_{i}=t f_{i} \cdot \log \frac{N}{n_{i}}$

$t f_{i}=$ the term frequency of word $i$ in the document

$N=$ the total number of sentences

$n_{i}=$ the number of sentences in which word $i$ occurs

This feature can be calculated as follows.

$$
\begin{aligned}
& \mathbf{f 3}=\frac{\sum_{i=1}^{k} W_{i}(S)}{\operatorname{Max}\left(\sum_{i=1}^{k} W_{i}\left(S_{i}^{N}\right)\right)} \\
& k=\text { number of words in sentence }
\end{aligned}
$$

\subsubsection{Sentence Length}

This feature is suitable when eliminating the sentences which are too short such as datelines or author names. The short sentences are not expected to belong to the summary.

$$
f 4=\frac{\text { Number of } \text { Words in Sentence }}{\text { Number of words occuring in longest sentence }}
$$

\subsubsection{Thematic Word}

This feature is related with domain specific words which occur frequently in a document are probably related topic. The score for this feature is calculated as the ratio of the number of thematic words that occur in the sentence over the maximum summary of thematic words in the sentence.

$$
f 5=\frac{\text { Number of thematic word in } S}{\text { Maximum }(\text { Number of Thematic Word })}
$$




\subsubsection{Proper Nouns}

Generally, the sentence that involves proper nouns is essential and it is most possibly contained in document summary. The score for this feature is counted as the ratio of the number proper nouns that occur in sentence over the sentence length the important sentence which contains maximum number of proper nouns.

$$
\text { f6 }=\frac{\text { Number of Proper nouns in } S}{\text { Sentence Length }}
$$

\subsubsection{Sentence to Sentence Similarity}

The cosine similarity formula gives the similarity between $\mathrm{S}$ and each other sentence. The term weight $\mathrm{w}_{\mathrm{i}}$ and $\mathrm{w}_{\mathrm{j}}$ of term $\mathrm{t}$ to $\mathrm{n}$ term in sentence $\mathrm{S}_{\mathrm{i}}$ and $\mathrm{S}_{\mathrm{j}}$ are represented as the vectors.

$$
\begin{aligned}
& \operatorname{Similarity}\left(S_{i}, S_{j}\right)=\frac{\sum_{t=1}^{N} W_{i t} W_{j t}}{\sqrt{\sum_{t=1}^{n} W_{i t}^{2}} \sqrt{\sum_{t=1}^{n} W_{j t}^{2}}} \\
& \mathbf{f 7}=\frac{\sum \operatorname{Similarity}\left(S_{i}, S_{j}\right)}{\operatorname{Maximum}\left(\sum \operatorname{Similarity}\left(S_{i}, S_{j}\right)\right)}
\end{aligned}
$$

\subsubsection{Numerical Data}

Generally, the sentence that involves numerical data is essential and it is most possibly contained in document summary. The score fort his feature is calculated as the ratio of the number of numerical data that occur in sentence over sentence length. Its score is calculated as[12]:

$$
\text { f8 }=\frac{\text { Number of Numerical Data in } S}{\text { Sentence Length }}
$$

\section{SUMMARIZATION METHODS}

\subsection{Query Based and Generic Summarization}

In query based text summarization the scoring of the sentences of a given document is based on the frequency counts of words or phrases. Higher scores are given to the sentences containing the query phrases rather than the ones with single query words. The sentences with highest scores are then extracted for the output summary together with their structural context. Portions of text may be extracted from different sections or subsections. The resulting summary is the union of such extracts. In the sentence extraction algorithm, whenever a sentence is selected for the inclusion in the summary, some of the headings in that context are also selected[8].

The query based sentence extraction algorithm[9] is as follows;

Algorithm:

1: Rank all the sentences according to their score.

2: Add the main title of the document to the summary.

3: Add the first level-1 heading to the summary.

4: While (summary size limit not exceeded)

5: Add the next highest scored sentence.

6: Add the structural context of the sentence: (if any and not already included in the summary)

7: Add the highest level heading above the extracted text (call this heading $\mathrm{h}$ ).

8: Add the heading before $h$ in the same level.

9: Add the heading after $h$ in the same level.

10: Repeat steps 7, 8 and 9 for the next highest level headings.

11: End while

In generic summaries are not query based[10]. Query based summaries are biased because they do not provide the overall review of the source document. They deal with the user queries only hence not suitable for content overview. To define the category of the document and to describe the main key points of the document generic summaries are required. A best generic summary considers the main topics of the documents and tries to minimize the redundancy as less as possible. 
Artificial intelligence (AI), deep learning, fuzzy logic and neural networks represent incredibly exciting and powerful machine learning-based techniques used to text summarization.

\subsubsection{Bayesian Classifier}

Given a training set of documents with hand-selected document extracts, develop a classification function that estimates the probability a given sentence is included in an extract. New extracts can then be generated by ranking sentences according to this probability and selecting a user-specified number of the top scoring ones.

For each sentence $s$ we compute the probability it will be included in a summary $S$ given the $k$ features which can be expressed using Bayes' rule as follows[22]:

$f_{j} ; j=1 . . . k$,

$P(s \in S \mid f 1, f 2, \ldots, f k)=\frac{P(f 1, f 2, \ldots, f k \mid s \epsilon S) P(s \in S)}{P(f 1, f 2, \ldots, f k)}$

Assuming statistical independence of the features:

$P(s \in S \mid f 1, f 2, \ldots, f k)=\frac{\prod_{j=1}^{k} P\left(f_{j} \mid s \in S\right) P(s \in S)}{\prod_{j=1}^{k} P(f j)}$

\subsubsection{Hidden Markov Model}

Hidden Markov Model(HMM)[25] is an another method to extract a sentence from a document. In contrast to a naive Bayesian approach, the Hidden Markov model has fewer assumptions of independence. In particular, the HMM does not assume that the probability that sentence $i$ is in the summary is independent of whether sentence $i-1$ is in the summary.

The main idea is using a sequential model to account for local dependencies between sentences. In HMM Model, three features were used:

- $\quad$ position of the sentence in the document,

- number of terms in the sentence,

- likeliness of the sentence terms given the document terms.

Figure 2: Hidden Markov Model to Extract Three Summary Sentences from a Document.

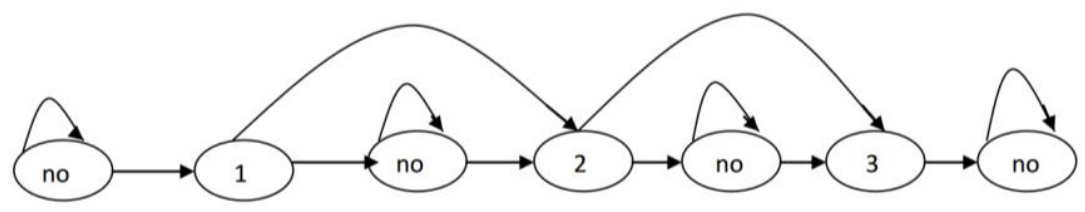

The HMM was structured as follows: it contained $2 \mathrm{~s}+1$ states, alternating between $s$ summary states and s+1 nonsummary states. The authors allowed "hesitation" only in nonsummary states and "skipping next state" only in summary states. Fig. 2. shows an example HMM with 7 nodes, corresponding to $\mathrm{s}=3$. Using the TREC dataset as training corpus, the authors obtained the maximum-likelihood estimate for each transition probability, forming the transition matrix estimate, whose element $(i, j)$ is the empirical probability of transitioning from state $i$ to $j$. Associated with each state $i$ was an output function, $(O)=\operatorname{Pr}(O \mid$ state $i)$ where $O$ is an observed vector of features. They made a simplifying assumption that the features are multivariate normal. The output function for each state was thus estimated by using the training data to compute the maximum likelihood estimate of its mean and covariance matrix. They estimated $2 \mathrm{~s}+1$ means, but assumed that all of the output functions shared a common covariance matrix. Evaluation was done by comparing with human generated extracts.

\subsubsection{Neural Networks Based Text Summarization}

Artificial Neural Networks(ANN) are one of the most popular and powerful classes of machine learning algorithms. ANN is used to generate summaries of arbitrary length news articles. A neural network is trained on a corpus of articles. The neural network is then modified, through fusion to produce a summary of the most ranked sentences of the article. Through feature fusion, the network discovers the importance of various features used to determine the Summary-worthiness of each sentence[16].

ANN has training and testing phases. In training phase, sentences in several test paragraphs where each sentence is identified as to whether it should be added in the summary or not. This is carried out by a human reader. The neural 
network learns the patterns which are inherent in sentences that should be added in the summary and those that should not be added.

The Neural Network has three layered feed-forward structure. It consists of seven input layer neurons, six hidden layer neurons and one output layer neurons. Each sentence is represented as a vector $[f 1, f 2, \ldots, f 7]$ which consists of 7 features. In this network, few features are different from before, but most of them are same. The features are selected according to position of document or position of the sentence.

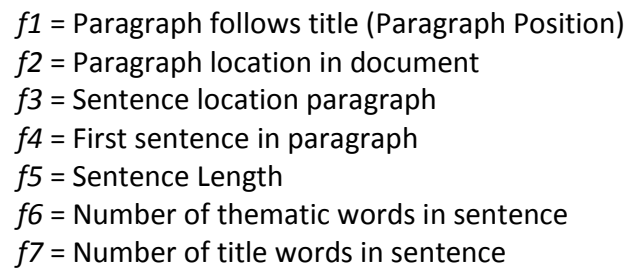

Figure 3: The Neural Network after Training

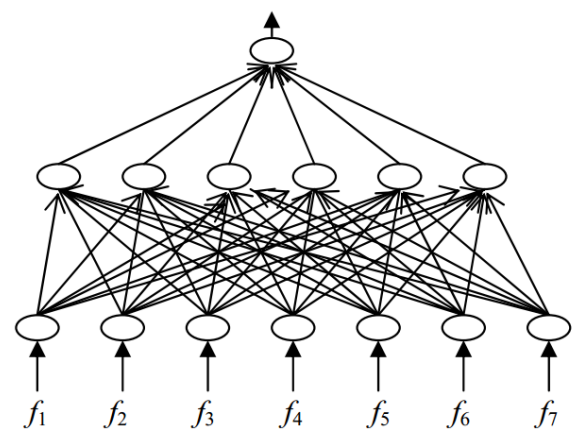

Text Summarization process consists of three phases: training, feature fusion and sentence selection. The first step involves training a neural network to recognize the type of sentences that should be added in the summary. In the second step, reduce the neural network and collapses the hidden layer unit activations into discrete values with frequencies. The third step, sentence selection, uses the modified neural network to filter the text and to select only the highly ranked sentences[17].

Figure 4: The neural Network after Pruning

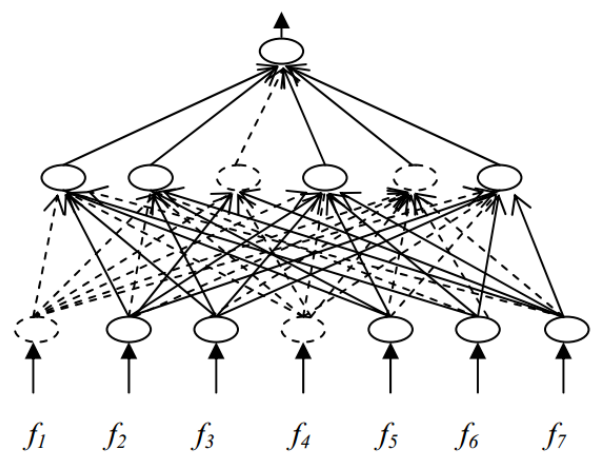

The hidden layer activation values for each children layer neuron are clustered utilizing an adaptive clustering technique. Each cluster is identified by its centroid and frequency. The activation value of each hidden layer neuron is replaced by the centroid of the cluster, which the activation value belongs to. This corresponds to collapsing the effect of common features. The combination of these two steps corresponds to generalizing the effects of features, as a whole, and providing control parameters for sentence ranking.

Another technique[18] which is using Neural Network for text summarizing add "Numerical Data Feture" to feature input list so their network uses 8 input neurons. After finding high ranked summary sentences by neural network we feed these sentences to rhetorical structure to find the discourse structure from that and find rhetorical relation in sentences which may help in finding better summary sentences, which further might be used to form better summary. 
Rhetorical Structure Theory[19] using rhetorical relations provide a methodical way for an analyst to analyse the text. An analysis is usually constructed by reading the text \& building a tree using the relations. The example given below is a title and summary, the original text, broke down into units having numbers, is:

\section{The Perception of Apparent Motion}

2. When the motion of an intermittently seen object is ambiguous

3. the visual system resolves confusion

4.by applying some tricks that reflect a bulletin knowledge of properties of the physical world.

Figure 5: Rhetorical Relations

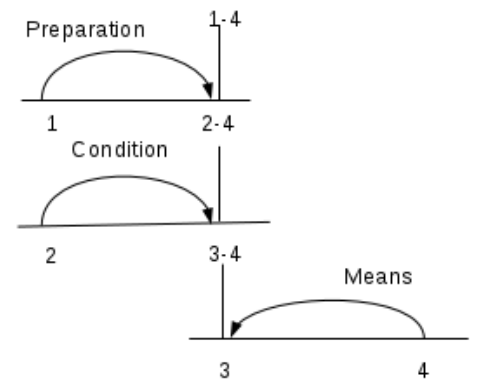

In the figure 3 number 1, 2, 3, 4 displaying the correspond units as explained above. 4th unit and 3rd unit forming a relation neans. 4th unit is the important part of this relation. So it is known as nucleus of the relation and 3rd unit is known as satellite of the relation. Similarly 2 nd unit to $3 \mathrm{rd}$ and 4 th unit is forming relation condition. spans may be composed of two or more units[18].

\subsubsection{Fuzzy Logic Based Text Summarization}

In this method, Fuzzy Logic rule and fuzzy logic set are used to extract the important sentences based on their features. Fuzzy logic techniques provide decision-support and expert systems with strong reasoning capabilities. Fuzzy logic set proposed by Zadeh[29] is a mathematical tool dealing with uncertainity, imprecision, vagueness and ambiguity. A few studies were done in text summarization. Witte and Bergle[30] present a fuzzy-theory based approach to coreference resolution and its application to text summarization. Automatic determination of coreference between noun phrases is fraught with uncertainty. They show how fuzzy sets can be used to design a new coreference algorithm which captures this uncertainty in an explicit way and allows us to define varying degrees of coreference. Patil and Kulkarni[31] also use Fuzzy Logic to score sentences after feauture selection and pre-processing step. They use eight features for text summarization: title word, sentence length, sentence position, numerical data, thematic words, sentence to sentence similarity, term weight and Proper Nouns.

The system involve in following steps:

- Perused the source archive into the system,

- In preprocessing step, the system extracts the individual sentences of the original documents. After, separate the input document into individual words. Next, remove stop words. The last step for preprocessing is word stemming;

- $\quad$ each sentence is related in vector of eight features that mentioned in Section II, whose values are obtained from the content of the sentence;

- $\quad$ the features are calculated to obtain the sentence score base on fuzzy logic method shows in Figure 6.;

- a set of highest score sentences are extracted as document summary based on the compression rate.

Fuzzy Logic System includes four components: Fuzzifier, Inference Engine, Defuzzifier and Fuzzy Knowledge Base. In the fuzzifier, crisp inputs are translated into linguistic values using a membership function to be used to the input linguistic variables. After fuzzification, the inference engine refers to the rule base containing fuzzy IFTHEN rules to derive the linguistic values. 


\section{Figure 6: Text Summarization Flowchart with Fuzzy Logic}

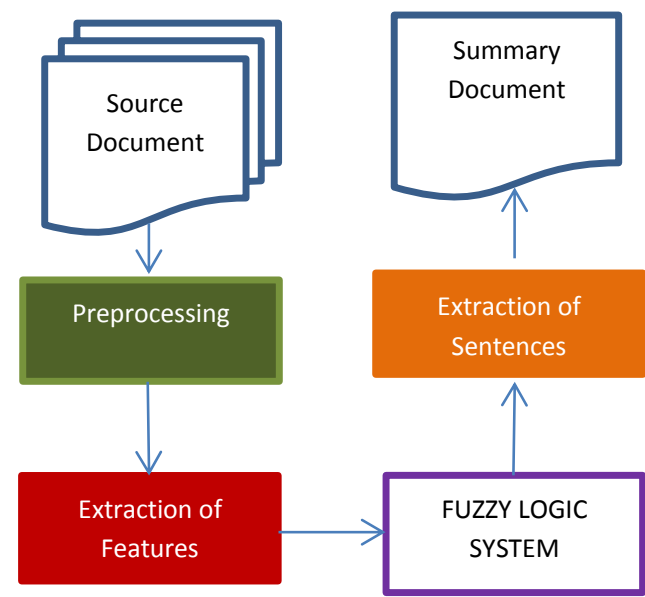

In the last step, the output linguistic variables from the inference are converted to the final crisp values by the defuzzifier using membership function for representing the final sentence score[12]. In order to implement text summarization based on fuzzy logic;

Thus each sentence is associated with 8 feature vector. Using all the 8 feature scores, the score for each sentence are derived using fuzzy logic method. The fuzzy logic method uses the fuzzy rules and triangular membership function .The fuzzy rules are in the form of IF-THEN. The triangular membership function fuzzifies each score into one of 3 values that is LOW, MEDIUM \& HIGH. Then we apply fuzzy rules to determine whether sentence is unimportant, average or important. This is also known as defuzzification. For example IF (F1is H) and (F2 is M) and (F3 is H) and (F4 is M) and (F5 is M) and (F6 is $\mathrm{M})$ and (F7 is $\mathrm{H}$ ) and (F8 is $\mathrm{H}$ ) THEN (sentence is important).

In sentence selection phase, all the sentences of a document are ranked in a descending order based on their scores. Top $\mathrm{n}$ sentences of highest score are extracted as document summary based on compression rate. Finally the sentences in summary are arranged in the order they occur in the original document.

\section{EVALUATION}

Evaluation methods are necessary to compare results between text summarization methods. Two main criterion for evaluating the proficiency of a system is precision and recall which are used for specifying the similarity between the summary which is generated by the system versus the one generated by human. The terms are defined in the following equation[23].

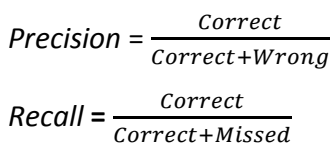

In these formula;

Correct $=$ the number of sentences that are the same in both summary which are generated by the system and human.

Wrong = the number of sentences presented in summary and produced by system but is not included in the Summary generated by human.

Precision $=$ the number of suitable sentences which are extracted by the system and Recall specify the number of suitable sentences that the summarization system missed.

Compression $\operatorname{Ratio}(C R)=\frac{\text { Length }(S)}{\text { Length }(T)}$

Retention $\operatorname{Ratio}(R R)=\frac{\text { Information in } S}{\text { Information in } R}$

The ROUGE, a set of metrics called Recall-Oriented Understudy for Gisting Evaluation, evaluation toolkit [28] that has become standards of automatic evaluation of summaries. It compares the summaries generated by the program with the human-generated (gold standard) summaries. For comparison, it uses n-gram statistics[27]. 


\section{CONCLUSION}

Owing to fast growth of technology and use of Internet, there is information overload. This problem can be solved if there are strong text summarizers which produces a summary of document to help user. In the last decades, Automatic Text Summarization is a popular research area and attracts a lot of attentions from different science branches. In this paper, we discuss type of summarization methods which might be used in a system to produce summary. Text summarization methods can be classified into extractive and abstactive summarization. An extractive summary is selection of important sentences from the original text. Abstractive Summariy is a method for novel phrasing describing the content of the text which requires heavy machinery from natural language processing, including grammars and lexicons for parsing and generation. Especially, this paper is focussing on extractive summarization methods.

Extractive bases summarization methods Bayesian Classifier, Hidden Markov Model, Neural Networks and Fuzzy Logic have to an extent, succeeded in making an effective summary of a document.

\section{REFERENCES}

[1] E. H. Hovy, Automated Text Summarization. The Oxford Handbook of Computational Linguistics, Chapter 32, pages 583-598. Oxford University Press, 2005.

[2] I. Mani, D. House, G. Klein, The TIPSTER SUMMAC Text Summarization Evaluation. In Proceedings of EACL, 1999.

[3] H. P. Luhn, "The automatic creation of literature abstracts", IBM Journal of Research and Development, vol. 2, pp. 159-165, 1958.

[4] U. Hahn, \& I. Mani, "The challenges of automatic summarization" IEEE-Computer, 33(11), 29-36, 2000.

[5] V. Gupta, G.S. Lehal, "A survey of Text Summarization Extractive Techniques”, Journal of Emerging Technologies in Web Intelligence, Vol. 2, No.3, August 2010.

[6] S.A. Babar, P. D. Patil, "Improving Performance of Text Summarization", International Conference on Information and Communication Technologies, ICICT, 2014.

[7] Sherry, P. Bhatia, "A Survey to Automatic Summarization Techniques", International Journal of Engineering Research and General Science Volume 3, Issue 5, September-October, 2015.

[8] S. Karmakar, T. Lad, H. Chothani, A Review Paper on Extractive Techniques of Text Summarization, International Research Journal of Computer Science (IRJCS) Issue 1, Volume 2, 2015.

[9] F. C. Pembe and T. Güngör, "Automated Query-biased and Structure-preserving Text Summarization on Web Documents," in Proceedings of the International Symposium on Innovations in Intelligent Systems and Applications, İstanbul, June 2007.

[10] G. Yihong, X. Liu. "Generic text summarization using relevance measure and latent semantic analysis." Proceedings of the 24th annual international ACM SIGIR conference on Research and development in information retrieval, ACM, 2001.

[11] S. Alfayoumy, J. Thoppil, A Survey of Unstructured Text Summarization Techniques, International Journal of Advanced Computer Science and Applications, Vol. 5, No. 4, 2014.

[12] L. Suanmali, N. Salim, M. S. Binwahlan, "Fuzzy Logic Based Method for Improving Text Summarization", International Journal of Computer Science and Information Security, Vol. 2, No. 1, 2009.

[13] P.Priya, G. and K Duraiswamy, "An Approach for Text Summarization Using Deep Learning Algorithm”, Journal of Computer Science, 19, 2014.

[14] Amy J.C. Trappey, Charles V. Trappey, "An R\&D knowledge management method for patent document summarization" Industrial Management \& Data Systems, vol.108. pp. 245-257. 2008.

[15] D. Ravichandran, P. Pantel, and E.H. Hovy, "Randomized Algorithms and NLP: Using Locality Sensitive Hash Functions for High Speed Noun Clustering", Proceedings of the conference of the Association for Computational Linguistics (ACL), 2005.

[16] Khosrow Kaikhah, "Automatic Text Summarization with Neural Networks", in Proceedings of Second International Conference on intelligent systems, IEEE, 40-44, Texas, USA, June 2004.

[17] Khosrow Kaikhah, "Text Summarization using Neural Networks", Departments of Faculty Publications Computer Science, Texas State University, 2004.

[18] Sarda A.T., Kulkarni A.R., "Text Summarization using Neural Networks and Rhetorical Structure Theory", International Journal of Advanced Research in Computer and Communication Engineering, 2015.

[19] W. C. Mann, S. Thompson, Rhetorical Structure Theory: Toward a functional theory of text organization. Text, 8 (3), 1988. 
[20] F.Kyoomarsi, H. Khosravi, E. Eslami and P.H. Dehkordy, "Optimizing Text Summarization Based on Fuzzy Logic", In proceedings of Seventh IEEE/ACIS International Conference on Computer and Information Science, IEEE, University of Shahid Bahonar Kerman, UK, 347$352,2008$.

[21] S.A.Babar, S.A.Thorat, "Improving Text Summarization using Fuzzy Logic \& Latent Semantic Analysis",International Journal of Innovative Research in Advanced Engineering (IJIRAE), 2014.

[22] J. Kupiec, J. Pedersen, and F. Chen, "A trainable document summarizer", In Proceedings of the 18th ACMSIGIR Conference, pages 6873, 1995 .

[23] E. Lahari, D.V.N.S. Kumar, M.Ubale, "A Comprehensive Survey on Feature Extraction in Text Summarization", E Padma Lahari et al , Int.J.Computer Technology \& Applications, Vol 5 (1),248-256, 2014.

[24] P.D. Patil, P.M. Mane, "An Overall Survey of Extractive Based Automatic Text Summarization Methods", International Journal of Science and Research (IJSR) 2014.

[25] J. Conroy, D.P. O'leary, “Text Summarization via Hidden Markov Models and Pivoted QR Matrix Decomposition", Proceedings of the 24th annual international ACM SIGIR conference on Research and development in information retrieval 2001.

[26] C. Cığır, M. Kutlu, İ. Çiçekli, "Generic Text Summarization for Turkish", The 24th International Symposium on Computer and Information Sciences, ISCIS 2009, 14-16 September 2009.

[27] C.Y. Lin, E.H. Hovy, "Automatic evaluation of summaries using n-gram co-occurrence statistics", Proceedings of HLT-NAACL-2003, Edmenton, Canada, 2003.

[28] C.Y. Lin, "ROUGE: A Package for Automatic Evaluation of Summaries" In Proceedings of Workshop on Text Summarization of ACL, Spain. 2004.

[29] L. Zadeh, “Fuzzy sets. Information Control” vol. 8, pp.338-353.1965.

[30] R. Witte and S. Bergler, "Fuzzy coreference resolution for summarization" In Proceedings of 2003 International Symposium on Reference Resolution and Its Applications to Question Answering and Summarization (ARQAS) 2003.

[31] P.D. Patil, N.J. Kalkurni, "Text Summarization Using Fuzzy Logic", International Journal of Innovative Research in Advanced Engineering (IIIRAE) Volume 1 Issue 3, 2014.

[32] S. Gholamrezazadeh, M. Salehi, B. Gholamzadeh "A Comprehensive Survey on Text Summarization Systems", Computer Science and its Applications, 2009. 